\title{
Numerical Modeling of the Dielectric Barrier Discharges Plasma Flow
}

\author{
Azizi Ahmadi, Jane Labadin, Phang Piau \\ Department of Computational Science \& Mathematics, \\ Faculty of Computer Science and Information \\ Technology, Universiti Malaysia Sarawak, \\ Sarawak, Malaysia. \\ azizi0407@gmail.com, ljane@fit.unimas.my, \\ pphang@fit.unimas.my
}

\begin{abstract}
Dielectric Barrier Discharge (DBD) is a discharge phenomenon where a high voltage is applied on at least two electrodes separated by an insulating dielectric material. Dielectric Barrier Discharge plasma actuator has been studied widely in this last decade but mostly the study is focusing on experimental research rather than mathematical modeling. The limitation with studying DBD plasma actuator experimentally is that it does not obtain direct information on the physics of the plasma flow, which is important in determining its efficiency. In this paper, we model the steady fluid model DBD plasma actuator mathematically. The preliminary result of the model are presented and discussed. To initiate the modeling process, the stream-function and vorticity are defined so that the Navier-Stokes momentum equation could be transformed into vorticity equation. The resulting two governing equations, which are vorticity and stream-function equations are solved numerically to obtain the vorticity of the flow in $x$ and $y$ directions. Finite difference method was adopted to discretize both equations and the system of equations is solved by the Gauss-Seidel method. Our numerical solutions show that the applied voltage plays an important role in the model. We found that as the applied voltage increases, the vorticity of the plasma flow also increases.
\end{abstract}

Keywords - Dielectric Barrier Discharges; Mathematical Modeling; Plasma Actuator

\section{INTRODUCTION}

Dielectric Barrier Discharges (DBD) is a discharge phenomenon where a high voltage is applied on at least two electrodes and the electrodes are insulated by at least one dielectric material. This situation will produce plasma discharges. This study is focusing on the plasma discharges that are produced by applying high voltage on the electrodes. This study is called Electrohydrodynamics or EHD. From the word itself, Electrohydrodynamics is a study of the dynamics of electrical charged fluid. From this definition, it is known that there are two things that need to be considered which are the electrical field and the fluid flow.

DBD plasma actuator has been studied widely in this last decade but mostly the study is focusing on experimental research (for example, [1] - [5]) rather than mathematical

\author{
Andrew R. H. Rigit \\ Department of Mechanical \& Manufacturing \\ Engineering, \\ Faculty of Engineering, Universiti Malaysia Sarawak \\ Sarawak, Malaysia. \\ arigit@feng.unimas.my
}

modeling. The limitation with studying DBD plasma actuator experimentally is it does not obtain direct information on the physics of the plasma flow, which is important in order to increase the efficiency of the plasma actuator itself [6].

In order to model DBD mathematically, there are two models that need to be formulated and decoupled it so that we can simulate the DBD's phenomena; electrostatics model [7] and fluid dynamics model. In this paper, we will present formulation of the fluid flow aspects of the DBD plasma actuator mathematically and the preliminary result of the model will be presented and discussed.

\section{MathEmatical Modeling}

As mentioned earlier, two models need to be developed in order to represent the whole DBD plasma actuator system, which are electrostatics model and fluid dynamics model. Electrostatics model is needed to obtain the body force that is required in the fluid dynamics model (see [8] - [10]). The electrostatics model has already been derived in our earlier work [7]. Therefore, this paper will mainly deal with the formulation of the fluid dynamics model.

Consider a Navier-Stokes momentum equation that describes the fluid movement substance, as follows:

$$
\frac{\partial \vec{v}}{\partial t}+(\vec{v} \cdot \nabla) \vec{v}=-\frac{1}{\rho} \nabla p+\mu \nabla^{2} \vec{v}+\vec{f}_{b}
$$

where $\vec{v}=$ flow velocity, $\rho=$ fluid density,$p=$ pressure , $\mu=$ viscosity,$\vec{f}_{b}=$ body force vector.

Since the aim of the modeling process is to obtain the vorticity of the fluid model then to do this the Navier-Stokes momentum equation is transformed into vorticity equation. We started-off by introducing the stream function, $\psi$, as:

$$
u=\frac{\partial \psi}{\partial y}, v=-\frac{\partial \psi}{\partial x},
$$

where the velocity of fluid is expressed by the components $u$ in the $x$ direction and $v$ in the $y$ direction. Thus, the relationship between the vorticity, $\vec{w}$, and the stream function, $\psi$ is 


$$
\begin{aligned}
\vec{w} & =\nabla \cdot \vec{v} \\
& =-\frac{\partial^{2} \psi}{\partial y^{2}}-\frac{\partial^{2} \psi}{\partial x^{2}}
\end{aligned}
$$

To initiate the transformation, the curl of both sides of equation (1) is written as:

$$
\nabla \times\left(\frac{\partial \vec{v}}{\partial t}+(\vec{v} \cdot \nabla) \vec{v}=-\frac{1}{\rho} \nabla p+\mu \nabla^{2} \vec{v}+\vec{f}\right) .
$$

On expanding equation (4) and simplifying it, we obtain

$$
\frac{\partial \vec{w}}{\partial t}+u_{y} \frac{\partial w}{\partial x}+v_{x} \frac{\partial w}{\partial y}=\frac{1}{\operatorname{Re}}\left(\frac{\partial^{2} w}{\partial y^{2}}+\frac{\partial^{2} w}{\partial x^{2}}\right)+\frac{\partial f^{x}}{\partial y}-\frac{\partial f^{y}}{\partial x}
$$

where $\operatorname{Re}$ is the Reynold's number, and $f^{x}$ and $f^{y}$ are body forces in $x$ and $y$ directions, respectively, as provided in [7].

For a steady flow, $\frac{\partial \vec{w}}{\partial t}=0$ holds. Hence, we obtain the vorticity equation,

$$
u_{y} \frac{\partial w}{\partial x}+v_{x} \frac{\partial w}{\partial y}=\frac{1}{\operatorname{Re}}\left(\frac{\partial^{2} w}{\partial y^{2}}+\frac{\partial^{2} w}{\partial x^{2}}\right)+\frac{\partial f^{x}}{\partial y}-\frac{\partial f^{y}}{\partial x} .
$$

Now, rewriting equation (3) gives us

$$
\frac{\partial^{2} \psi}{\partial y^{2}}+\frac{\partial^{2} \psi}{\partial x^{2}}=-\vec{w}
$$

so that we have the governing equation for the streamfunction. Thus, the system of equations (6) and (7) forms the plasma flow model for the DBD.

\section{BOUNDARY CONDITIONS}

This system of equations (equations (6) and (7)) has two unknown variables which are the vorticity, $\vec{w}$ and stream function, $\psi$, and together with the boundary conditions and initial value for the body force vector (as mentioned in [7]) are then solved numerically.

\begin{tabular}{|c|c|}
\hline \multicolumn{1}{|c|}{$\vec{f}_{b}=0$} \\
\hline Electrode & Dround \\
\hline \multicolumn{2}{|c|}{ Dielectric } \\
\hline \multicolumn{2}{|c|}{} \\
\hline \multirow{2}{*}{$\vec{f}_{b}=0$} & G \\
\hline
\end{tabular}

Figure 1. Configuration of the Dielectric Barrier Discharges

The initial values and the boundary conditions for the vorticity, stream-function and velocity flow, are kept zero at the outer boundary, on the dielectric material, and on the electrodes. The body force obtained from [2] is only applied on the dotted line portion as shown in Figure 1, whereas at other parts, we assume no body force acting on the system.

\section{NUMERICAL FORMULATION}

We adopt the finite centered difference method to discretize both equations (6) and (7). Equation (7) is best suit second-order centered difference method, as follows:

$$
\frac{\psi_{i+1, j}-2 \psi_{i, j}+\psi_{i-1, j}}{\Delta x^{2}}+\frac{\psi_{i, j+1}-2 \psi_{i, j}+\psi_{i, j-1}}{\Delta y^{2}}=-w_{i, j}
$$

For equation (6), the convective terms are discretized by using first-order backward difference method whereas the diffusive terms are discretized by using first-order centered difference method, as shown below.

$$
\begin{aligned}
& \frac{u_{i, j+1}-u_{i, j}}{\Delta y} \frac{w_{i+1, j}-w_{i, j}}{\Delta x}+\frac{v_{i+1, j}-v_{i, j}}{\Delta x} \frac{w_{i, j+1}-w_{i, j}}{\Delta y}= \\
& \frac{1}{\operatorname{Re}}\left(\frac{w_{i, j+1}-2 w_{i, j}+w_{i, j}-1}{\Delta y^{2}}+\frac{w_{i+1, j}-2 w_{i, j}+w_{i-1, j}}{\Delta x^{2}}\right)+ \\
& \frac{f_{i, j+1}^{x}-f_{i, j-1}^{x}}{2 \Delta y}-\frac{f_{i+1, j}^{y}-f_{i-1, j}^{y}}{2 \Delta x}
\end{aligned}
$$

Equations (8) and (9) are then rearranged into

$$
\begin{aligned}
& 2\left(\Delta y^{2}+\Delta x^{2}\right) \psi_{i, j}=\Delta x^{2} \Delta y^{2} w_{i, j}+\Delta y^{2} \psi_{i+1, j} \\
& +\Delta y^{2} \psi_{i-1, j}+\Delta x^{2} \psi_{i, j+1}+\Delta x^{2} \psi_{i, j-1} \\
& G_{i, j} w_{i, j}=A_{i+1, j} w_{i+1, j}+B_{i, j+1} w_{i, j+1}+ \\
& C_{i-1, j} w_{i-1, j}+D_{i, j-1} w_{i, j-1}+\text { force }
\end{aligned}
$$

where

$$
\begin{aligned}
A_{i+1, j} & =\frac{\operatorname{Re} \Delta x\left(u_{i, j+1}-u_{i, j}\right)-\Delta y}{\operatorname{Re} \Delta x^{2} \Delta y} \\
B_{i, j+1} & =\frac{\operatorname{Re} \Delta y\left(v_{i+1, j}-v_{i, j}\right)-\Delta x}{\operatorname{Re} \Delta y^{2} \Delta x} \\
C_{i-1, j} & =-\frac{1}{\operatorname{Re} \Delta x^{2}} \\
D_{i, j-1} & =-\frac{1}{\operatorname{Re} \Delta y^{2}} \\
G_{i, j} & =\frac{\operatorname{Re} \Delta x \Delta y\left(u_{i, j+1}-u_{i, j}+v_{i+1, j}-v_{i, j}\right)-2 \Delta x^{2}-2 \Delta y^{2}}{\operatorname{Re} \Delta x^{2} \Delta y^{2}} \\
\text { force } & =\frac{f_{i, j+1}^{x}-f_{i, j-1}^{x}-\frac{f^{y}-f^{y}{ }_{i-1, j}}{2 \Delta y}}{2 \Delta x}
\end{aligned}
$$

